\title{
The role of the Born approximation in nonlinear inversion
}

\author{
Roel Snieder \\ Department of Theoretical Geophysics, University of Utrecht, PO Box 80.021, 3508 TA \\ Utrecht, The Netherlands
}

Received 12 May 1989, in final form 7 November 1989

\begin{abstract}
A perturbation analysis of nonlinear inversion is presented. As a prototype of nonlinear inversion, inverse scattering with the Marchenko equation is considered from a perturbative point of view. It is shown that inverse scattering methods using the Marchenko equation implicitly reconstruct the potential by removing the nonlinearities from the data, and by performing a Born inversion of the resulting linear component in the data (the first Born approximation). This is illustrated with a one-dimensional example. This interpretation of the mechanism of inverse scattering algorithms clarifies the 'miracle of Newton', and has profound consequences for both the theoretical and the practical aspects of inverse scattering, in particular for the stability of inverse scattering schemes. As shown in several examples, the arguments presented for inverse scattering with the Marchenko equation can be generalised to a wide class of nonlinear inverse problems.
\end{abstract}

\section{Introduction}

Inverse scattering methods such as the Marchenko or the Gel'fand-Levitan algorithms belong to the rare examples of an exact formulation of a nonlinear inverse problem. Despite the simple mathematical formulation of these algorithms, surprisingly little is known about the mechanism by which these algorithms actually perform the inversion. Which characteristics of the data are actually used for the reconstruction of the unknown potential? How are the nonlinearities in the problem handled? This paper clarifies these issues by showing a perturbative analysis of exact inverse scattering methods.

This pertubative analysis is presented for the one-dimensional (ID) Marchenko equation, which forms the solution for the inverse problem for the 1D plasma wave equation (Balanis 1972, Burridge 1980). However, the obtained results depend only on the general structure of the Marchenko equation, but not on its details. This means that the results of this paper can be generalised to any nonlinear inversion scheme for which the forward problem has a regular perturbation expansion, and for which the inverse problem can be formulated in terms of operators that act repeatedly on the data. The main conclusion of the perturbative analysis is that only the linear components of the data (the first Born approximation, or the single reflected waves) contribute to the reconstruction of the potential. Implicitly the nonlinear components are subtracted from the data by solving the integral equation of inverse scattering.

This observation gives new insights in nonlinear inversion algorithms. It makes it easy to understand why inverse scattering methods suffer from stability problems (Koehler and Taner 1977), why exact inverse scattering methods for media with a variable wave velocity have only been formulated relative to a fast reference medium 
(Cheney et al 1989), and it gives an example of the remarkable 'miracle of Newton' for inverse scattering in three dimensions.

The 1D plasma wave equation and the Marchenko equation are presented in section 2. In the subsequent section a perturbative analysis is applied to the Marchenko equation. This is illustrated in section 4 with a numerical example. Section 5 features three examples of a perturbative analysis of other nonlinear inversion schemes (the 'miracle' of Newton, the inversion of Corones et al (1983) using invariant embedding, and the iterative inversion of Morawetz and Kriegsmann (1983)). In an appendix it is shown explicitly that the quadratic nonlinearities for the 3D Schrödinger equation are correctly subtracted from the scattering data by the Newton-Marchenko algorithm.

\section{Inverse scattering for the 1D plasma wave equation}

The perturbative analysis of inverse scattering algorithms is the same for all inverse scattering schemes which involve the solution of an integral equation similar to the Marchenko equation or the Gel'fand-Levitan equation. Rather than giving a general derivation, the perturbation analysis is presented for the inversion of the $1 \mathrm{D}$ plasma wave equation (PWE) using the Marchenko equation. The arguments presented in this paper are easily generalised for other inverse scattering equations such as presented by Rose et al (1986).

The problem is considered where the wavefield $u(x, t)$ satisfies the PwE:

$$
u_{x, x}(x, t)-u_{t t}(x, t)-V(x) u(x, t)=0
$$

where $V(x)$ is an unknown potential. For simplicity it is assumed that

$$
V(x)=0 \quad \text { for } x<0 .
$$

Furthermore it is assumed that $V \in L^{2}$ and that (Chadan and Sabatier 1989):

$$
\int_{0}^{\infty}(1+|x|)|V(x)| \mathrm{d} x<\infty .
$$

This potential is being probed by an impulsive wave coming in from the left, so that:

$$
u(x, t)=\delta(t-x) \quad \text { for } t<0 .
$$

The waves reflected by the potential are recorded at $x=0$, so that the data for the inversion are given by the reflection time series $R(t)$ :

$$
R(t)=u(x=0, t)-\delta(t) .
$$

The inverse problem consists of the determination of the unknown potential $V(x)$ given the reflections $R(t)$.

The solution of this inverse problem has been formulated both in the spectral domain (Agranovich and Marchenko 1963) and in the time domain (Balanis 1972, Burridge 1980 ). The reflection time series $R(t)$ serves as an integral kernel in the Marchenko equation:

$$
K(x, t)+R(x+t)+\int_{-i}^{x} K(x, \tau) R(\tau+t) \mathrm{d} \tau=0 .
$$

In shorthand notation this equation is also written as

$$
K+R+K R=0 .
$$


Once $K(x, t)$ has been determined from the Marchenko equation, one can find the potential using

$$
V(x)=2 \frac{\mathrm{d} K(x, x)}{\mathrm{d} x} .
$$

\section{A perturbative analysis for the forward and inverse problem of the 1D plasma wave equation}

In order to understand the role of the nonlinearities in inverse scattering it is necessary to distinguish the linear effects from the nonlinear effects. For this reason it is convenient to attach a coupling parameter $\varepsilon$ to the potential:

$$
V=\varepsilon V(x) .
$$

A perturbation expansion of the forward problem can be obtained by expressing the solution of (1) with the incident wave (3) as the following integral equation:

$$
u(x, t)=\delta(t-x)+\varepsilon \int \mathrm{d} x^{\prime} \int \mathrm{d} t^{\prime} G_{0}\left(x, t ; x^{\prime}, t^{\prime}\right) V\left(x^{\prime}\right) u\left(x^{\prime}, t^{\prime}\right)
$$

with $G_{0}$ the causal Green function of the unperturbed PWE:

$$
\partial_{x x} G_{0}\left(x, t ; x^{\prime}, t^{\prime}\right)-\partial_{t t} G_{0}\left(x, t ; x^{\prime}, t^{\prime}\right)=\delta\left(x-x^{\prime}\right) \delta\left(t-t^{\prime}\right) .
$$

This Green function is given by

$$
G_{0}\left(x, t ; x^{\prime}, t^{\prime}\right)=-\frac{1}{2} H\left(t-t^{\prime}-\left|x-x^{\prime}\right|\right)
$$

$H(t)$ being the Heaviside function.

Iterating the integral equation (9) leads to a Neumann series which can be considered as a Taylor expansion in $\varepsilon$. Considering the resulting Neumann series for $x=0$ leads with equation (5) to a perturbation expansion for the reflected waves:

$$
R(t)=\varepsilon R_{1}(t)+\varepsilon^{2} R_{2}(t)+\ldots
$$

the $R_{n}$ being given by:

$$
\begin{aligned}
& R_{1}(t)=\int \mathrm{d} x_{1} \mathrm{~d} t_{1} G_{0}\left(x=0, t ; x_{1}, t_{1}\right) V\left(x_{1}\right) \delta\left(t_{1}-x_{1}\right) \\
& R_{2}(t)=\int \mathrm{d} t_{1} \mathrm{~d} t_{1} \mathrm{~d} x_{2} \mathrm{~d} t_{2} G_{0}\left(x=0, t ; x_{1}, t_{1}\right) V\left(x_{1}\right) G_{0}\left(x_{1}, t_{1} ; x_{2}, t_{2}\right) \delta\left(t_{2}-x_{2}\right) \\
& R_{3}(t)=\int \mathrm{d} x_{1} \mathrm{~d} t_{1} \mathrm{~d} x_{2} \mathrm{~d} t_{2} \mathrm{~d} x_{3} \mathrm{~d} t_{3} G_{0}\left(x=0, t ; x_{1}, t_{1}\right) V\left(x_{1}\right) \\
& \quad \times G_{0}\left(x_{1}, t_{1} ; x_{2}, t_{2}\right) V\left(x_{2}\right) G_{0}\left(x_{2}, t_{2} ; x_{3}, t_{3}\right) V\left(x_{3}\right) \delta\left(t_{3}-x_{3}\right)
\end{aligned}
$$

with obvious higher-order generalisations. The term $R_{1}(t)$ is the part of the data which depends linearly on the potential (the single reflected waves). In analogy with the quantum mechanical nomenclature this term will be referred to as the Born approximation. $R_{2}(t)$ describes the wave that experienced two interactions with the potential, etc. 
Just as the reflection $R(t)$, the solution $K(x, t)$ of the Marchenko equation (5) is also a nonlinear function of the potential; this function can also be expanded in a Taylor series in $\varepsilon$ :

$$
K(x, t)=\varepsilon K_{1}(x, t)+\varepsilon^{2} K_{2}(x, t)+\ldots
$$

Inserting the Taylor expansions (12) and (14) in the Marchenko equation (6), and equating the coefficients of equal powers of $\varepsilon$ one obtains a hierarchy of equations for $K_{n}$ :

$$
\begin{aligned}
& K_{1}=-R_{1} \\
& K_{2}=-R_{2}-K_{1} R_{1} \\
& K_{3}=-R_{3}-K_{1} R_{2}-K_{2} R_{1} \\
& K_{n}=-R_{n}-\sum_{m=1}^{n-1} K_{m} R_{n-m} .
\end{aligned}
$$

These equations constitute the relation between the $K_{n}$ and the terms $R_{n}$ in the Neumann series. A recursive application of these relations allows for the elimination of the $K_{n}$ from the right-hand side of $(15 d)$. This gives

$$
\begin{aligned}
& K_{1}=-R_{1} \\
& K_{2}=-R_{2}+R_{1} R_{1} \\
& K_{3}=-R_{3}+R_{2} R_{1}+R_{1} R_{2}-R_{1} R_{1} R_{1} \\
& K_{n}=\sum_{m=1}^{n}(-1)^{m} \sum_{i_{1}+\cdots+i_{m}=n} R_{i_{1}} \ldots R_{i_{m}} .
\end{aligned}
$$

For example, $K_{2}(x, x)$ is given explicitly by

$$
K_{2}(x, x)=-R_{2}(2 x)+\int_{-x}^{x} R_{1}^{2}(x+\tau) \mathrm{d} \tau .
$$

Alternatively, one can insert (8) and the Taylor expansion (14) in the relation (7) between $V$ and $K$. Equating the coefficients of equal powers of $\varepsilon$ gives

$$
\begin{aligned}
& 2 \frac{\mathrm{d} K_{1}}{\mathrm{~d} x}(x, x)=V(x) \\
& 2 \frac{\mathrm{d} K_{n}}{\mathrm{~d} x}(x, x)=0 \quad n \geqslant 2 .
\end{aligned}
$$

The above expressions imply that $V(x)$ is completely determined by $K_{1}$. According to $(15 a)$, the first-order contribution $K_{1}(x, x)$ depends only on the first Born approximation $R_{1}$, and is thus independent of the nonlinear components in the data. This means that only the first Born approximation contributes to the reconstruction of the potential, and that the nonlinear components in the data do not contribute to the reconstruction of the potential.

So what happens to the nonlinear components $R_{n}(n \geqslant 2)$ in the data? As an example, consider the quadratic term $K_{2}(x, x)$ in equation $(15 b)$. This term consists of the difference between the second Born term $R_{2}$, and the iterated term $K_{1} R_{1}$. According to $(17 b), K_{2}$ does not give a net contribution to the reconstruction of the potential, which 
means that the second Born approximation $R_{2}$ is being subtracted from the data by the iterated term $K_{1} R_{1}$. It can be seen explicitly in (16e) that the second-order Born term $R_{2}$ is being cancelled by an iteration of the first Born approximation $R_{1}$. The same mechanism is operative for the higher-order terms. For example, the third Born approximation $R_{3}$ is being cancelled in $(16 c)$ by repeated iterations of the lower-order Born approximations $R_{1}$ and $R_{2}$.

This means that in the inversion using the Marchenko equation, only the first Born approximation contributes to the reconstruction of the potential, and that in the inversion the nonlinearities are being subtracted from the data. Note that this does not imply that one need only perform a Born inversion of the data in order to obtain the potential. The reason for this is that one does not measure the first Born approximation; the data consist of a sum of both the linear components and the nonlinear components. One really needs to go through a nonlinear inversion, such as the Marchenko method, in order to eliminate the effect of the nonlinearities.

One can show from the integral representation for $K(x, t)$ (Burridge 1980) that $K(0,0)=0$. With $(17 a, b)$ this implies that, for $x>0$,

$$
K_{n}(x, x)=0 \quad n \geqslant 2 \text {. }
$$

\section{A numerical example}

In this section a numerical example is presented to show that the nonlinear components are correctly subtracted from the data by using the Marchenko equation. In these examples the potential shown in figure 1 is used. The precise form of the potential is not important, but it should be kept in mind that the potential consists essentially of two scattering zones, and that waves with wavelengths much shorter than the distance between the scattering zones can bounce back and forth between the two sides of the potential. The low-frequency components in the wavefield interact in a simultaneous way with both sides of the potential.

The first three terms $(13 a-c)$ of the Neumann series are shown together with their sum in figure 2. The Born approximation $R_{1}$ consists of reflections with opposite polarity from the two sides of the potential. The third-order contribution $R_{3}$ shows a distinct

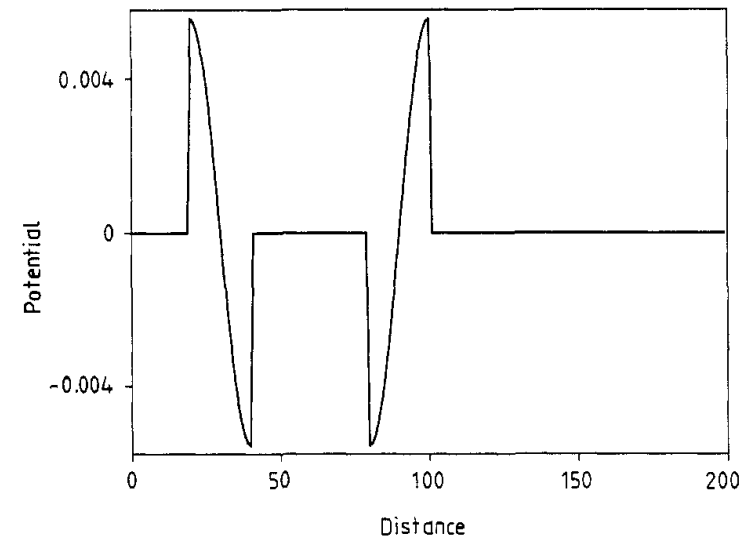

Figure 1. Potential used in the numerical example. 


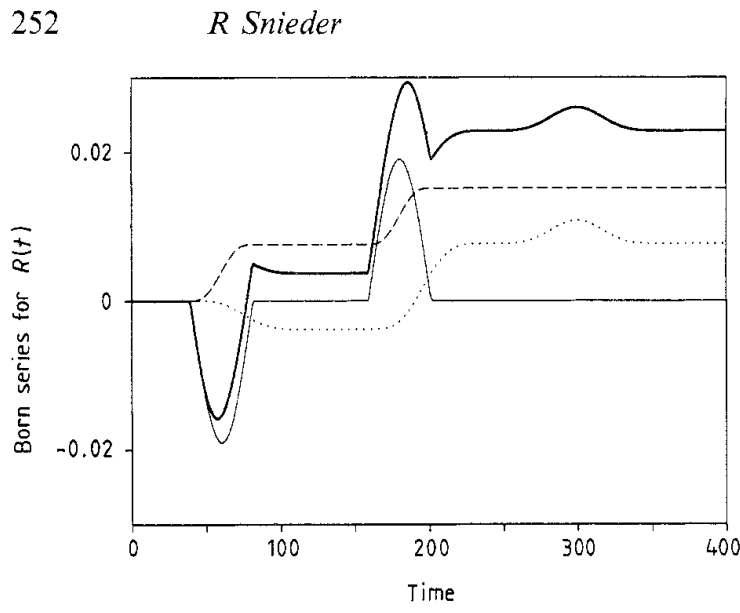

Figure 2. The first (thin full line), second (broken line) and third (dotted line) Born approximations and their sum (thick full line).

wave arrival around $t=280$, this is the wave that has bounced back and forth once between the sides of the potential. The static offset of the signals reflects the fact that no fixed boundary values are imposed on $u(x, t)$; this behaviour is the same as for an infinite stretched string without fixed boundary conditions.

The first-order contribution $K_{1}(x, x)$ computed with $(15 a)$ and $(13 a)$ is shown in figure 3. Application of the differentiation (17a) of this function leads to a result which is indistinguishable from the original potential of figure 1 . This reflects the fact that it is only the first Born approximation which gives a non-zero contribution to the reconstruction of the potential.

The second-order contributions to $K_{2}(x, x)$ computed with $(13 a, b)$ and $(15 b)$ are shown in figure 4 . The second Born approximation $R_{2}$ is exactly cancelled by the repeated iteration $K_{1} R_{1}\left(=-R_{1} R_{1}\right)$ of the first Born approximation, which verifies that the quadratic component in the data is correctly subtracted. Note that this does not mean that $K_{2}(x, t)$ vanishes for all $t \neq x$, since the condition (18) only implies that $K_{2}(x, x)$ vanishes. However, the latter condition is indeed satisfied.

Figure 5 features the cubic contributions to $K_{3}(x, x)$ computed with $(13 a-c)$ and $(15 c)$. Again it can be seen that the third-order Born approximation $R_{3}$ is being cancelled by repeated iterations of lower-order Born approximations. The total contribution to $K_{3}(x, x)$ is not quite zero; the slight deviation around $x=100$ is due to numerical errors. Note that the wave which has bounced back and forth between the

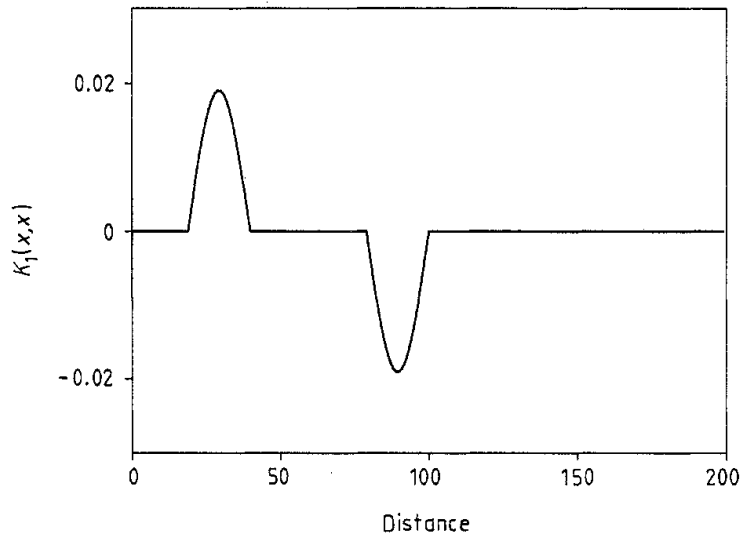

Figure 3. The first-order contribution $K_{1}(x, x)$ to the reconstruction of the potential. The derivative $2 \mathrm{~d} K_{1}(x, x) / \mathrm{d} x$ is indistinguishable from the true potential in figure 1 . 

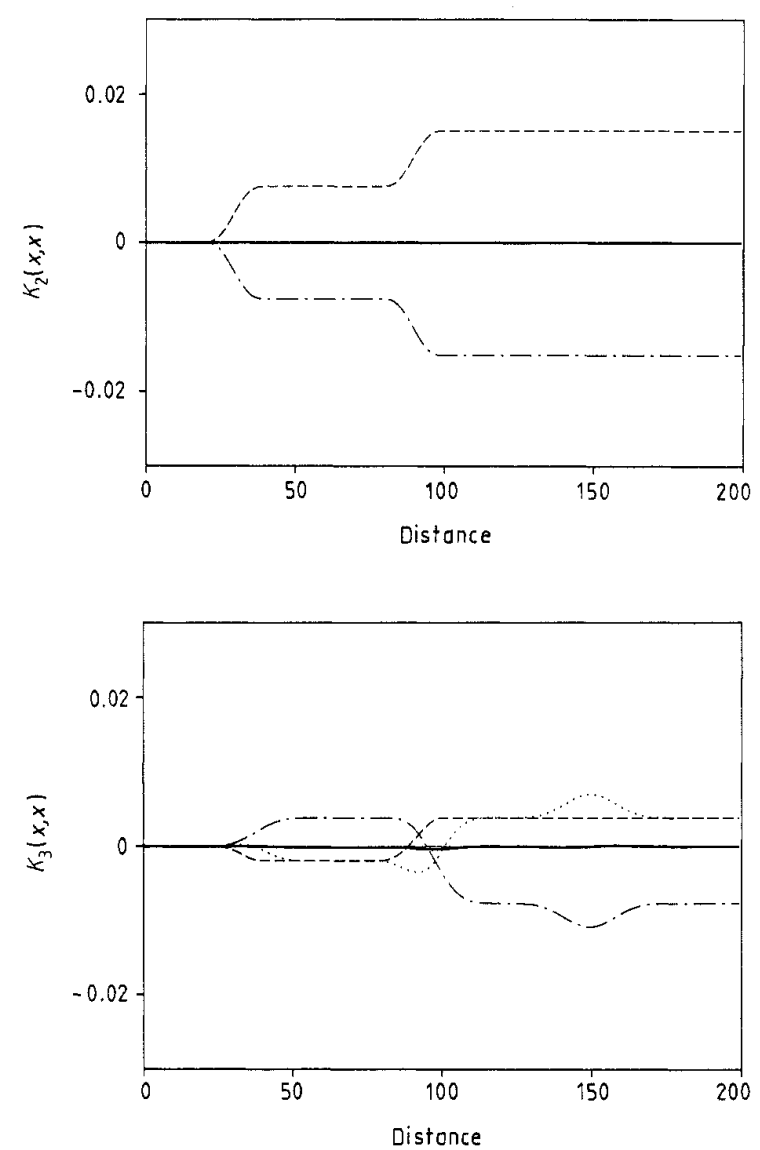

Figure 4. The second-order contributions $-R_{2}$ (chain line) and $-K_{1} R_{1}$ (broken line) to the reconstruction of the potential, with their sum $K_{2}(x, x)$ (full line).

sides of the potential (the valley around $x=150$ ) is eliminated so that it does not contribute to the reconstruction of the potential.

In practical inversions one does not, of course, known which parts of the data are due to linear effects, and which parts are caused by nonlinearities. Therefore one should really solve the Marchenko equation, or use some other nonlinear inversion scheme. As noted by Ge (1987), the Marchenko equation can efficiently be solved by iteration. In this algorithm the starting value is

$$
\tilde{K}^{(1)}(x, t)=-R(x, t)
$$

and the following iterations are defined by

$$
\tilde{K}^{(n+1)}(x, t)=-R(x, t)-\int_{-t}^{x} \tilde{K}^{(n)}(x, \tau) R(\tau+t) \mathrm{d} \tau .
$$

If this process converges, the final solution satisfies the Marchenko equation $(6 a)$. It should be noted that $\tilde{K}^{(n)}$ and $K_{n}$ are fundamentally different functions, $K_{n}$ depends by definition (14) only on the potential in the $n$th power, whereas $\widetilde{K}^{n}$ contains scattering effects of different orders.

The algorithm of Ge (1987) applied to the scattering data for the 1D potential of figure 1 is shown in figure 6 . In the iterations shown only the terms up to third order are 


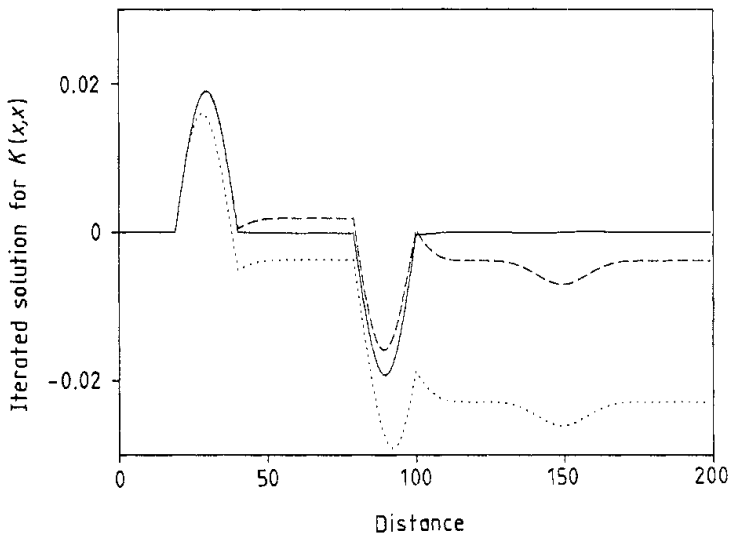

Figure 6. Iterative solution of the Marchenko equation after one (dotted line), two (broken line), and three iterations (full line). Only terms up to third order have been taken into account.

included. (For simplicity, $K(x, x)$ is shown rather than its derivative.) After the first iteration, the left bump of the potential (around $x=30$ ) is already reconstructed quite well, but the second bump (around $x=80$ ) and the area to the right are poorly reconstructed. The reason for this is that the first iteration of the Marchenko equation is equivalent to doing a Born inversion of the full data including the nonlinearities. The effect of the nonlinearities becomes stronger in the later parts of the signal (because then the waves have had time to bounce back and forth), so that after the first iteration the reconstruction suffers most from incorrectly treated nonlinearities further to the right. However, in subsequent iterations these incorrectly handled nonlinearities are subtracted from the reconstruction. The reconstruction after three iterations gives, after differentiation, exactly the potential. In this simple example, three iterations were sufficient to reconstruct the true potential because only terms up to third order were taken into account. However, when higher-order nonlinearities are present in the data one has to perform more iterations.

It is possible to show that after $n$ iterations all the multiple scattering effects up to $n$th order are correctly removed from the reconstructed potential, and that the error is of order $\varepsilon^{n+1}$. In order to see this, insert the Neumann series (12) in the algorithm $(19 a, b)$; this gives, in the abbreviated notation of section 3 ,

$\tilde{K}^{(1)}=-\varepsilon R_{1}-\varepsilon^{2} R_{2}-\varepsilon^{3} R_{3}+\mathrm{O}\left(\varepsilon^{4}\right)$

$\tilde{K}^{(2)}=-\varepsilon R_{1}-\varepsilon^{2}\left(R_{2}-R_{1} R_{1}\right)-\varepsilon^{3}\left(R_{3}-R_{1} R_{2}-R_{2} R_{1}\right)+\mathrm{O}\left(\varepsilon^{4}\right)$

$\tilde{K}^{(3)}=-\varepsilon R_{1}-\varepsilon^{2}\left(R_{2}-R_{1} R_{1}\right)-\varepsilon^{3}\left(R_{3}-R_{1} R_{2}-R_{2} R_{1}+R_{1} R_{1} R_{1}\right)+\mathrm{O}\left(\varepsilon^{4}\right)$.

Using equation (16) one finds that

$$
\begin{aligned}
& \tilde{K}^{(1)}=\varepsilon K_{1}+\mathrm{O}\left(\varepsilon^{2}\right) \\
& \tilde{K}^{(2)}=\varepsilon K_{1}+\varepsilon^{2} K_{2}+\mathrm{O}\left(\varepsilon^{3}\right) \\
& \tilde{K}^{(3)}=\varepsilon K_{1}+\varepsilon^{2} K_{2}+\varepsilon^{3} K_{3}+\mathrm{O}\left(\varepsilon^{4}\right)
\end{aligned}
$$

with obvious generalisations to higher order. Applying the differentiations (17a) and $(17 b)$ one finds that

$$
2 \frac{\mathrm{d} \tilde{K}^{(n)}(x, x)}{\mathrm{d} x}=\varepsilon V(x)+\mathrm{O}\left(\varepsilon^{n+1}\right) .
$$


This means that after $n$ iterations, all nonlinear scattering effects up to $n$th order are correctly subtracted from the data and do not contribute anymore to the reconstructed potential. The number of iterations needed in practice depends on the degree of nonlinearity and the required accuracy. For example, with seismic reflection data one can sometimes clearly identify a set of multiply reflected waves. If the $N$ th multiple is of the same order as the noise level, one needs to perform $N$ iterations.

\section{Generalisations to other nonlinear inversion methods}

The preceding theory was developed for the 1D plasma wave equation. However, the arguments employed for the perturbation analysis of the forward and the inverse problem did not rely in an essential way on the details of the PWE or the Marchenko equation. The only essential ingredient in the analysis was that the forward problem could be expressed as a regular series expansion in $\varepsilon$, and that the inversion could be expressed in the form of operators that act repeatedly on the data. A formal proof of this statement can be found in Snieder (1990), where a perturbation analysis is applied to nonlinear inversion in general. Rather than repeating that proof, three examples are presented in the following subsections of well known nonlinear inverse scattering problems for which only the first Born approximation contributes to the reconstruction of the inhomogeneity, and where the nonlinearities are removed in the inversion.

\subsection{Example 1. Inverse scattering for the 3D Schrödinger equation and the 'miracle of Newton'}

An inverse scattering algorithm for the three-dimensional Schrödinger equation has been formulated by Newton $(1980,1981)$. In this method, one needs to solve an integral equation similar to the Marchenko equation (6). Just as in the one-dimensional case, the integral kernel for this integral equation depends on the scattering data. The potential follows by a differentiation of the solution of the integral equation. Therefore, the arguments of the preceding sections apply equally well to the Newton-Marchenko method.

The Schrödinger equation in three dimensions is given by

$$
\left(\nabla^{2}+k^{2}\right) \psi(k, \hat{\boldsymbol{n}}, \boldsymbol{r})-\varepsilon V(\boldsymbol{r}) \psi(k, \hat{\boldsymbol{n}}, \boldsymbol{r})=0 .
$$

It is assumed that $V \in L^{2}$ and that positive numbers $C$ and $a$ exist, such that

$$
|V(\boldsymbol{r})| \leqslant C(a+|\boldsymbol{r}|)^{-\mu} \quad|\nabla V(\boldsymbol{r})| \leqslant C(a+|\boldsymbol{r}|)^{-\gamma}
$$

with $\mu>3$ and $v>7 / 2$ (Chadan and Sabatier 1989). If the potential is irradiated with an incident plane wave, the wavefunction in the far field is asymptotically given by:

$$
\psi(k, \hat{\boldsymbol{n}}, \boldsymbol{r})=\exp (\mathrm{i} k \hat{\boldsymbol{n}} \cdot \boldsymbol{r})+A_{k}(\hat{\boldsymbol{r}}, \hat{\boldsymbol{n}}) \frac{\exp (\mathrm{i} k r)}{r}
$$

$A_{k}$ being the scattering amplitude. The unit vector $\hat{n}$ denotes the direction of the incoming plane wave with wavenumber $k$.

The Newton-Marchenko method proceeds by computing for every point $\boldsymbol{r}$, for every incident $\hat{\boldsymbol{n}}$ and every final $\hat{\boldsymbol{n}}^{\prime}$, an integral kernel $R$ from the scattering data $A_{k}$ :

$$
R\left(\alpha, \hat{\boldsymbol{n}}, \hat{\boldsymbol{n}}^{\prime}, \boldsymbol{r}\right)=\frac{\mathrm{i}}{4 \pi^{2}} \int_{-\infty}^{\infty} \mathrm{d} k k A_{k}\left(-\hat{\boldsymbol{n}}^{\prime}, \hat{\boldsymbol{n}}\right) \exp \left\{-\mathrm{i} k\left[\alpha+\left(\hat{\boldsymbol{n}}+\hat{\boldsymbol{n}}^{\prime}\right) \cdot \boldsymbol{r}\right]\right\} .
$$


This integral kernel is then used in the Newton--Marchenko equation

$K(\alpha, \hat{\boldsymbol{n}}, \boldsymbol{r})=\int \mathrm{d}^{2} n^{\prime} R\left(\alpha, \hat{\boldsymbol{n}}, \hat{\boldsymbol{n}}^{\prime}, \boldsymbol{r}\right)+\int_{0}^{\infty} \mathrm{d} \beta \int \mathrm{d}^{2} n^{\prime} R\left(\alpha+\beta, \hat{\boldsymbol{n}}, \hat{\boldsymbol{n}}^{\prime}, \boldsymbol{r}\right) K\left(\beta, \hat{\boldsymbol{n}}^{\prime}, \boldsymbol{r}\right)$.

In this section and the appendix, $\int \mathrm{d}^{2} n^{\prime}$ denotes an integration over the unit sphere. The potential is finally recovered by differentiation:

$$
V(\boldsymbol{r})=-2 \hat{\boldsymbol{n}} \cdot \nabla K(\alpha=0, \hat{\boldsymbol{n}}, \boldsymbol{r}) .
$$

There are two aspects of the Newton-Marchenko equation which are not well understood. First, the right-hand side of equation (28) depends on the unit vector $\hat{\boldsymbol{n}}$, whereas the left-hand side is independent of $\hat{\boldsymbol{n}}$. This is called the 'miracle of Newton.' Equation (28) should be true if $A_{k}$ is indeed caused by scattering by a local potential. However, for general functions $A_{k}$, the miracle need not necessarily be satisfied (i.e. the right-hand side of (28) depends on $\hat{n}$ and in this case no local potential exists but a non-local potential exists when equation (28) still has a solution).

Second, there is the paradox that the Newton-Marchenko equation requires scattering data for all directions of incidence, for all scattering directions, and for all energies. However, for a Born inversion it suffices to measure a more restricted set of scattering data. (For example, it can be inferred from ( $30 a$ ) that a Born inversion of the potential can be performed by using the scattering amplitude for only one angle of incidence, all energies and all scattering directions.) It therefore appears that the Newton-Marchenko method requires a redundant data set. Note that this redundancy argument applies to the Born inversion, but not necessarily to the nonlinear inversion. The point of view of nonlinear inversion presented in this paper sheds light on both paradoxes. It is therefore instructive to do a perturbative analysis of the Newton-Marchenko method. This analysis is analogous to the analysis presented in section 3 for the one-dimensional PWE.

The scattering amplitude can be expanded in the following Born series (Rodberg and Thaler 1967):

$$
A_{k}\left(\hat{\boldsymbol{n}}^{\prime}, \hat{\boldsymbol{n}}\right)=\sum_{n=1}^{\infty} A_{k}^{(n)}\left(\hat{\boldsymbol{n}}^{\prime}, \hat{\boldsymbol{n}}\right) \varepsilon^{n}
$$

with

$$
\begin{aligned}
& A_{k}^{(1)}\left(\hat{\boldsymbol{n}}^{\prime}, \hat{\boldsymbol{n}}\right)=\frac{-1}{4 \pi} \int \mathrm{d}^{3} r \exp \left(-\mathrm{i} k \hat{\boldsymbol{n}}^{\prime} \cdot \boldsymbol{r}\right) V(\boldsymbol{r}) \exp (\mathrm{i} k \hat{\boldsymbol{n}} \cdot \boldsymbol{r}) \\
& \boldsymbol{A}_{k}^{(2)}\left(\hat{\boldsymbol{n}}^{\prime}, \hat{\boldsymbol{n}}\right)=\frac{1}{16 \pi^{2}} \int \mathrm{d}^{3} r \int \mathrm{d}^{3} \boldsymbol{r}^{\prime} \exp \left(-\mathrm{i} k \hat{\boldsymbol{n}}^{\prime} \cdot \boldsymbol{r}\right) V(\boldsymbol{r}) \frac{\exp \left(\mathrm{i} k\left|\boldsymbol{r}-\boldsymbol{r}^{\prime}\right|\right)}{\left|\boldsymbol{r}-\boldsymbol{r}^{\prime}\right|} V\left(\boldsymbol{r}^{\prime}\right) \exp \left(\mathrm{i} k \hat{\boldsymbol{n}} \cdot \boldsymbol{r}^{\prime}\right) .
\end{aligned}
$$

The fact that it is assumed that the scattering amplitude can be written as the Born series for a local potential, implies that the analysis presented in this section has no bearing on the characterisation problem; it only has implications for the construction problem. The solution $K$ from the Newton-Marchenko equation (27) is implicitly a function of the potential and can be expanded in a Taylor series as in equation (14). The first-order contribution $K_{1}$ follows by applying the equivalent of (15a) to the Newton-Marchenko equation (27):

$$
K_{1}(\alpha, \hat{\boldsymbol{n}}, \boldsymbol{x})=\int \mathrm{d}^{2} n^{\prime} R_{1}\left(\alpha, \hat{\boldsymbol{n}}, \hat{\boldsymbol{n}}^{\prime}, x\right),
$$


where $R_{1}$ is obtained by inserting the first Born approximation (30a) in (26). This gives, after carrying out the $\hat{n}^{\prime}$ integration,

$K_{1}(\alpha, \hat{\boldsymbol{n}}, \boldsymbol{x})=\frac{1}{4 \pi^{2} \mathrm{i}} \int_{-\infty}^{\infty} \mathrm{d} k \int \mathrm{d}^{3} r V(\boldsymbol{r}) \frac{\sin (k|\boldsymbol{r}-\boldsymbol{x}|)}{|\boldsymbol{r}-\boldsymbol{x}|} \exp \{\mathrm{i} k[\hat{\boldsymbol{n}} \cdot(\boldsymbol{r}-\boldsymbol{x})-\alpha]\}$.

Putting $\alpha=0$ and performing the $k$ integral leads to

$K_{1}(\alpha=0, \hat{n}, \boldsymbol{x})=\frac{1}{4 \pi} \int \mathrm{d}^{3} r \frac{V(\boldsymbol{r})}{|\boldsymbol{r}-\boldsymbol{x}|}[\delta(|\boldsymbol{r}-\boldsymbol{x}|-\hat{\boldsymbol{n}} \cdot(\boldsymbol{r}-\boldsymbol{x}))-\delta(|\boldsymbol{r}-\boldsymbol{x}|+\hat{\boldsymbol{n}} \cdot(\boldsymbol{r}-\boldsymbol{x}))]$.

Replacing the integration variable $\boldsymbol{r}$ by $\boldsymbol{r}+\boldsymbol{x}$, switching to polar coordinates and carrying out the integration over the angles gives

$$
K_{1}(\alpha=0, \hat{n}, \boldsymbol{x})=\frac{1}{4} \int_{0}^{\infty} \mathrm{d} r(V(\boldsymbol{x}+r \hat{\boldsymbol{n}})-V(\boldsymbol{x}-r \hat{\boldsymbol{n}}))
$$

so that

$$
-2 \hat{\boldsymbol{n}} \cdot \nabla_{x} K_{1}(\alpha=0, \hat{\boldsymbol{n}}, \boldsymbol{x})=-\frac{1}{2} \hat{\boldsymbol{n}} \cdot \int_{0}^{\infty} \mathrm{d} r\left(\nabla_{x} V(\boldsymbol{x}+r \hat{\boldsymbol{n}})-\nabla_{x} V(\boldsymbol{x}-r \hat{\boldsymbol{n}})\right) .
$$

In this expression $\nabla_{x}$ denotes the gradient with respect to the $x$ coordinates. Introducing the vector $\boldsymbol{y}=r \hat{n}$, and converting the $\boldsymbol{x}$ differentiations to $\boldsymbol{y}$ differentiations gives

$$
-2 \hat{\boldsymbol{n}} \cdot \nabla_{x} K_{1}(\alpha=0, \hat{\boldsymbol{n}}, \boldsymbol{x})=-\frac{1}{2} \int_{0}^{A x} \mathrm{~d} \boldsymbol{y} \cdot\left(\nabla_{y} V(\boldsymbol{x}+\boldsymbol{y})+\nabla_{y} V(\boldsymbol{x}-\boldsymbol{y})\right) .
$$

Since the potential is assumed to vanish at infinity, this gives

$$
-2 \hat{\boldsymbol{n}} \cdot \nabla_{x} K_{1}(\alpha=0, \hat{\boldsymbol{n}}, \boldsymbol{x})=V(\boldsymbol{x})
$$

which confirms the occurrence of the miracle (28). The right-hand side of (36) is indeed independent of $\hat{n}$, the reason being that the potential only gives a contribution in the point $\boldsymbol{y}=0$ (which is independent of $\hat{\boldsymbol{n}}$ ) and in the points $\boldsymbol{y}= \pm \hat{\boldsymbol{n}} \infty$ (where the potential vanishes). In the derivation we can thus see the miracle 'in action' in a situation where the miracle (28) should hold.

In an analogous way to $(17 b)$, the higher-order terms $K_{n}(n \geqslant 2)$ satisfy $-2 \hat{\boldsymbol{n}} \cdot \nabla_{x} K_{n}(\alpha=0, \hat{\boldsymbol{n}}, \boldsymbol{x})=0$, so that these terms do not contribute to the reconstruction of the potential. This relation is explicitly verified for the quadratic terms in the appendix. The first iteration of the first Born approximation is thus the only term which contributes to the reconstruction of the potential; this means that the mechanism by which the miracle occurs in the construction of the potential is explained by the above derivation. However, note that it is assumed in (30) that the data $A_{k}$ are the Born series of some local potential. If this is not the case the argument of this section breaks down, and the miracle need not occur. The treatment of this section does not address the characterisation problem, which is a critical issue for the occurrence of the miracle.

The problem of the apparent redundancy of the Newton-Marchenko method can also be understood with the results of this paper. It is true that the Newton-Marchenko method requires more data than are needed for a Born inversion. However, according to the results of section 3 , two steps are implicitly taken in nonlinear inversion. The nonlinearities are removed from the data, and a Born inversion of the linear components of the data is performed. It is conceivable that the removal of the nonlinearities requires more data than are needed for a Born inversion of the linear component in the data. The 
argument that the Newton-Marchenko algorithm requires more data than a Born inversion does not necessarily imply that it requires a redundant data set, because it is possible that the removal of the nonlinearities requires a larger data set than is needed for a Born reconstruction of the potential.

\subsection{Example 2. Nonlinear inversion using invariant embedding}

An alternative to the inverse scattering methods using integral equations is formulated using Ricatti equations (Gjevik et al 1976, Corones et al 1983, Bregman et al 1985). Corones et al (1983) consider reflected waves recorded at $x=0$ for the following 1D equation:

$$
u_{x x}-u_{t t}+\varepsilon V(x) u_{x}=0 .
$$

It is assumed that the potential extends from $x=0$ to $x=X$. They analyse this equation using an invariant embedding approach where ones studies a suite of truncated potentials extending from an arbitrary point $x$ to the right side $X$ of the true potential. The reflected waves $R(x, t)$ then depends on the position $x$ of the left point of truncation. For details the reader is referred to Corones et al (1983) who show that $R(x, t)$ satisfies the following Ricatti equation

$$
\partial_{x} R(x, t)-2 \partial_{t} R(x, t)=-\frac{1}{2} \varepsilon V(x) \int_{0}^{t} R(x, s) R(x, t-s) \mathrm{d} s
$$

with boundary conditions

$$
\begin{aligned}
& R(x, 0)=-\frac{1}{4} \varepsilon V(x) \\
& R(X, t)=0 .
\end{aligned}
$$

For the forward problem one specifies $V(x)$, and hence by virtue of $(40 a), R(x, 0)$, and one integrates (39) leftwards towards $x=0$ to obtain the reflected waves for the untruncated medium:

$$
r(t)=R(0, t)
$$

In the inverse problem one specifies $r(t)$, and hence $R(0, t)$, and one integrates (39) rightwards towards $x=X$. The potential follows then from (40a). The forward and inverse problem thus constitute a mapping from the $x$ axis to the $t$ axis and vice versa, using the function $R(x, t)$ as an intermediary.

In order to facilitate a perturbation expansion of the forward problem one needs to integrate (39) and it is useful to use expression (22) of Corones et al (1983):

$R\left(x_{1}, t-2 x_{1}\right)-R\left(x_{0}, t-2 x_{0}\right)=-\frac{1}{2} \varepsilon \int_{x_{0}}^{x_{1}} \mathrm{~d} s \int_{0}^{t-2 s} \mathrm{~d} \tau V(s) R(s, \tau) R(s, t-2 s-\tau)$.

For the forward problem one needs to integrate leftwards; therefore take $x_{1}<x_{0}$. Setting $t=2 x_{0}$, and using $(40 a)$ one finds after a rearrangement of variables that:

$$
R(x, t)=-\frac{1}{4} \varepsilon V(x+t / 2)+\frac{1}{2} \varepsilon \int_{x}^{x+t / 2} \mathrm{~d} s \int_{0}^{t+2(x-s)} \mathrm{d} \tau V(s) R(s, \tau) R(s, 2 x-2 s+t-\tau) .
$$


$R(x, t)$ can be expressed as a Taylor series in $\varepsilon$, the different powers $\varepsilon^{n}$ indicating the $n$ th-order scattering effects:

$$
R(x, t)=\varepsilon R_{1}(x, t)+\varepsilon^{2} R_{2}(x, t)+\varepsilon^{3} R_{3}(x, t)+\ldots .
$$

Inserting this expansion in (43) one obtains, equating the coefficients of equal powers of $\varepsilon$,

$$
\begin{aligned}
& R_{1}(x, t)=-\frac{1}{4} V(x+t / 2), \\
& R_{2}(x, t)=0 \\
& R_{3}(x, t)=\frac{1}{2} \int_{x}^{x+t / 2} \mathrm{~d} s \int_{0}^{t+2(x-s)} \mathrm{d} \tau V(s) R_{1}(s, \tau) R_{1}(s, 2 x-2 s+t-\tau) .
\end{aligned}
$$

The $R_{n}(x, t)$ for even orders $n$ vanish because only the reflected wavefield is considered. The reflected waves $r(t)$ for the full medium from $x=0$ to $x=X$ have an expansion similar to (44). Setting $x=0$ in (45), and inserting (45a) in (45c) one finds for the non-zero terms $r_{1}$ and $r_{3}$ :

$$
\begin{aligned}
& r_{1}(t)=-\frac{1}{4} V(t / 2) \\
& r_{3}(t)=\frac{1}{32} \int_{0}^{t / 2} \mathrm{~d} s \int_{0}^{t-2 s} \mathrm{~d} \tau V(s) V(s+\tau / 2) V(t / 2-\tau / 2) .
\end{aligned}
$$

In these expressions $r_{1}$ describes the single reflected waves, and $r_{3}$ describes the waves that are reflected three times. The generalisations to higher orders is straightforward.

For the inverse problem, one needs to integrate rightward from $x=0$. In order to do so set $x_{0}=0$ in (42); using (41) this gives, after a redefinition of variables,

$R(x, t)=r(t+2 x)-\frac{1}{2} \varepsilon \int_{0}^{x} \mathrm{~d} s \int_{0}^{t+2(x-s)} \mathrm{d} \tau V(s) R(s, \tau) R(s, 2 x-2 s+t-\tau)$.

Iterating this equation once, and using ( $46 a$ ) to eliminate the potential, gives for the firstand third-order effects

$R(x, t)=r(t+2 x)+2 \varepsilon \int_{0}^{x} \mathrm{~d} s \int_{0}^{t+2(x-s)} \mathrm{d} \tau r(2 s) r(\tau+2 s) r(2 x+t-\tau)$.

The estimated model $\tilde{V}(x, \varepsilon)=-4 R(x, 0)$ follows from this expression by setting $t=0$. Inserting the expansions of $R(x, t)$ and $r(t)$ in powers of $\varepsilon$ in this expression one finds

$\tilde{V}(x, \varepsilon)=-4 \varepsilon r_{1}(2 x)-4 \varepsilon^{3} r_{3}(2 x)-8 \varepsilon^{3} \int_{0}^{x} \mathrm{~d} s \int_{0}^{2(x-s)} \mathrm{d} \tau r_{1}(2 s) r_{1}(\tau+2 s) r_{1}(2 x-\tau)$.

It can be seen from (46a) and (46b) that the third-order scattering term $r_{3}(t)$ is being cancelled by the triple integral of the Born approximation $r_{1}(t)$. The only term contributing to the reconstruction of the potential is the first-order term $-4 \varepsilon r_{1}(2 x)$. According to (46a) this gives $\tilde{V}(x, c)=\varepsilon V(x)$, as it should. The extension of the derivation presented in this section to higher orders is tedious but straightforward.

\subsection{Example 3. The iterative inversion of Morawetz and Kriegsmann (1983)}

The inverse problem for the ID PWE (1) has been solved in an iterative way by Morawetz and Kriegsmann (1983). (A similar iterative inversion scheme for higher-dimensional problems was presented by Morawetz (1981).) In their formulation the potential is 
assumed to be an even function, $V(x)=V(-x)$, which vanishes for $|x|>x_{0}$. The incident wave also has even parity:

$$
u_{0}(x, t)=\delta(t-x)+\delta(t+x) .
$$

The reflected waves are recorded at $x=0$, and the data are given by

$$
R(t)=u_{x}(0, t)
$$

In the formulation of Morawetz and Kriegsmann (1983) a Jost solution $P(x, t)$ is used, which satisfies the PWE(1) with the boundary condition

$$
P(x, t)=\delta(t-x) \quad \text { for } x>x_{0} .
$$

By using a representation theorem which relates the data (at $x=0$ ) via the Jost solution to the wavefield just after the wavefront $(u(x, x))$, one can derive the following equation for the unknown potential (Morawetz and Kriegsmann 1983):

$$
\begin{aligned}
-\frac{1}{2} \varepsilon V(x)= & \int_{-\infty}^{\infty} \mathrm{d} s \frac{\mathrm{d}}{\mathrm{d} x}[P(x, x-s)-P(-x, x-s)] \\
& \times\left(P(0,-s)-\int_{0}^{\infty} S(t-s)\left(R\left(r^{\prime}\right)+2 \delta^{\prime}(t)\right) \mathrm{d} t\right)
\end{aligned}
$$

with

$$
S^{\prime}(t)=-P(0, t) \text {. }
$$

Applying an integration by parts to (53) and redefining

$$
r(t)=\int_{0}^{t} R\left(t^{\prime}\right) \mathrm{d} t^{\prime}
$$

one finds that

$$
\frac{1}{2} \varepsilon V(x)=\int_{-\infty}^{\infty} \mathrm{d} s \int_{0}^{x} \mathrm{~d} \tau B(x, s) P(0, \tau-s) r(\tau)
$$

with

$$
B(x, t)=\frac{\mathrm{d}}{\mathrm{d} x}[P(x, x-t)-P(-x, x-t)] .
$$

Equation (56) is a nonlinear integral relation for the potential, because the Jost solution $P(x, t)$ (and hence $B(x, t)$ ) is a nonlinear function of the potential, which enters the right-hand side quadratically. The data $r(t)$ also depend nonlinearly on the potential, although in practical inversions one considers the data of course as a fixed independent quantity.

A perturbative analysis can be applied to (56). In order to do so the following expansions in $\varepsilon$ can be used, these expansion can be derived from the Neumann series for the appropriate integral equations

$$
\begin{aligned}
& P(0, t)=P_{0}(0, t)+\varepsilon P_{1}(0, t)+\varepsilon^{2} P_{2}(0, t)+\ldots \\
& B(x, t)=B_{0}(x, t)+\varepsilon B_{1}(x, t)+\varepsilon^{2} B_{2}(x, t)+\ldots \\
& r(t)=\varepsilon r_{1}(t)+\varepsilon^{2} r_{2}(t)+\ldots
\end{aligned}
$$

The absence of the zeroth-order term for $r(t)$ can readily be inferred from $(50),(51)$ and (55). The zeroth-order terms $P_{0}$ and $B_{0}$ are independent of the potential. Inserting these 
expansions in (56) gives for the first- and second-order terms:

$$
\begin{aligned}
\frac{1}{2} \varepsilon V(x)= & \varepsilon \int_{-\infty}^{\infty} \mathrm{d} s \int_{0}^{\infty} \mathrm{d} \tau B_{0}(x, s) P_{0}(0, \tau-s) r_{1}(\tau) \\
& +\varepsilon^{2} \int_{-\infty}^{\infty} \mathrm{d} s \int_{0}^{\infty} \mathrm{d} \tau\left[B_{0}(x, s) P_{0}(0, \tau-s) r_{2}(\tau)\right. \\
& \left.+B_{0}(x, s) P_{1}(0, \tau-s) r_{1}(\tau)+B_{1}(x, s) P_{0}(0, \tau-s) r_{1}(\tau)\right] .
\end{aligned}
$$

Equating the coefficients from equal powers of $\varepsilon$, one sees that the potential depends only on $r_{1}(t)$; hence once more it is only the first Born approximation that contributes to the reconstruction of the potential. The term proportional to $\varepsilon^{2}$ on the right-hand side is zero, because there is no corresponding term on the left. This means that the double reflected waves $r_{2}(t)$ are being cancelled by repeated iterations of first-order quantities. This derivation is trivially extended to higher orders. This implies once more that the nonlinear components in the data do not contribute to the reconstruction of the potential, and that they are cancelled in the inversion by repeated iterations of lower-order terms.

\section{Discussion}

Inverse scattering algorithms construct implicitly the potential by subtracting the nonlinear components from the data, and performing a Born inversion of the linear component in the data. Although this point of view does not help us very much in the design of inversion schemes, it gives us some useful insights in the mechanism of nonlinear inversion. This conclusion pertains to any nonlinear problem where the forward problem can be expanded as a regular expansion, and where the inverse problem can be formulated in terms of a sum of operators which act repeatedly on the data (see Snieder 1990). The examples of section 5 illustrate this statement.

The fact that only the Born approximation contributes to the reconstruction of the potential does not imply that it suffices to perform a Born inversion of the data. The reason for this is that the data consist of the complete Neumann series instead of the first Born approximation. Removing the nonlinear components from the data is an essential ingredient of nonlinear inversion. This removal is achieved in an implicit fashion in any nonlinear inversion scheme that reconstructs the true potential.

The fact that inverse scattering algorithms subtract the nonlinearities from the data by iteration of an integral equation implies that there exist interrelations between the part of the data corresponding to different orders of nonlinearity. (If this were not the case, then it would be impossible to subtract the nonlinearities by repeated iterations of the lower-order nonlinearities.) These interrelations are currently poorly understood and are needed to obtain a better understanding of inverse scattering algorithms.

Errors in the data have a disastrous effect on the performance of inverse scattering algorithms. For example, it is shown by Koehler and Taner (1977) that timing errors lead to a severe instability of a Goupillaud inversion for a layered medium. (As shown by Berryman and Greene (1980), the Goupillaud inversion is equivalent to inverse scattering using the Marchenko equation (6).) The reason for this is now easy to understand. Errors in the data destroy the interrelations between the different nonlinear components in the data, so that the nonlinearities can no longer be subtracted properly. The data are 
especially sensitive to timing errors, because timing errors destroy the relation between the arrival times between single reflected waves and multiple reflected waves. The detrimental effect of data errors on nonlinear inversion is illustrated with a numerical example in Snieder (1990). In some nonlinear inversions this instability may be partially countered with some form of regularisation. In an optimisation approach, such as shown in Snieder and Tarantola (1989) for the imaging of quantum mechanical potentials, such a regularisation can be incorporated in a natural way.

The stability of inverse scattering algorithms has received considerable interest (Koehler and Taner 1977, Krueger 1981, Bregman et al 1985, Carrion 1986). The fact that the subtraction of nonlinear components in the data leads to instabilities in nonlinear inversion applies to a wide class of nonlinear inverse problems, such as those presented in section 5. The nonlinear inversion scheme in the examples of Krueger (1981) appears to be rather stable. However, the reflection coefficients in the example of Krueger (1981) are of the order of $5 \%$, so that one is for practical purposes in the linear domain. This is confirmed in figure 3 of Krueger (1981) which shows that increasing the data error by a factor 5 leads to a pointwise fivefold increase in the error of the reconstructed model, a typical linear phenomenon. In contrast, it is shown in Bregman et al (1985) that numerical truncation and round-off effects leads to instabilities if one is in the strongly nonlinear domain. Similarly, Koehler and Taner (1977) showed that multiply reflected waves lead to instabilities in the Goupillaud inversion of seismic reflection data. For a proper appraisal of the stability of a nonlinear inversion scheme, it is crucial that one is in the nonlinear domain.

Exact inverse scattering methods always rely on a causality principle (Rose et al 1984, 1985a,b, DeFacio and Rose 1985). Usually this causality principle manifests itself by the triangularity of some integral kernel. The interpretation of inverse scattering in this paper elucidates the crucial role of causality in nonlinear inversion. Causality implies that the linear component of the data arrives before the multiply scattered waves. For reflection data this is obvious, because a wave that is reflected only once arrives earlier than a wave that has bounced back and forth in the medium. For transmission data, the progressing wave expansion shows that the wavefield right after the direct wave depends linearly on the potential, and that the nonlinear components in the wavefield arrive later (Burridge 1980, Rose et al 1984). This means that if one wants to subtract the nonlinearities from the data at time $t_{0}$, one need only consider the signal at earlier times $t \leqslant t_{0}$. This is implicit in equations (15) where the nonlinearities are subtracted. For example, in (16d) the quadratic term $R_{2}$ at time $t=2 x$ is being cancelled by a combination of linear terms $R_{1}$ at earlier times $t \leqslant 2 x$. This causality principle also forms the basis of various kinds of stripping algorithms (e.g. Bojarski 1980, Krueger 1981, Bube and Burridge 1983, Bregman et al 1985).

Exact inverse scattering methods were formulated for the Schrödinger equation. In the Schrödinger equation, the propagation speed of the waves is not affected by the potential. The formulation of exact inverse scattering methods for media with a variable wave velocity is problematic. (In one-dimensional inverse problems with a variable velocity one usually transforms the velocity away by converting depth to travel time (e.g. Burridge 1980).) For three-dimensional problems with variable wave velocity, an integral equation for the inverse problem has been formulated by Rose et al (1985a) for the special case where the wave velocity in the inhomogeneity is less than or equal to the wave velocity in the surrounding reference medium. Cheney et al (1989) remove this limitation using an inverse scattering algorithm which relates the variable velocity medium to a fast reference medium in such a way that the scattered waves are always 
slower than the waves in the reference medium, so that the desired causality properties can be employed. The results of this paper allow a better understanding of the additional complications of exact inversion schemes for scattering media with a varying wave velocity. In inverse scattering, one deals with the nonlinearities by subtracting them from the data. In a medium with a fixed wave velocity, one can infer the arrival time of a multiply reflected wave if one knows the arrival times of the single reflected waves. This is no longer possible if the velocity is unknown because in that case one does not know at which time one should subtract the nonlinear components from the data. This problem is aggravated by the fact that for media with a variable wave velocity the nonlinear components in the data may arrive before the linear components in the data, which considerably complicates the inversion for media with a variable wave velocity. In fact, it is not known if the inverse problem for $3 \mathrm{D}$ media with an unrestricted variable wave velocity is well-posed (Cheney et al 1989).

As noted by Ge (1987), the second iteration of the Marchenko equation produces the same contribution to the reconstructed potential as the quadratic term of a perturbative inversion scheme for the Schrödinger equation derived by Moses (1956). It is shown by Snieder (1990) that the perturbative analysis of the Marchenko equation can be extended to any nonlinear inverse problem where both the forward and inverse problem can be expressed as regular Taylor series. It is therefore not surprising that the iterative solution of the Marchenko equation leads to the same algorithm as for a perturbative inversion as developed by Moses (1956). It follows from the fact that the arguments presented in this paper can be extended to a very wide class of nonlinear inverse problems (Snieder, 1990) that the subtraction of the nonlinearities and the reconstruction of the inhomogeneity by the linear component in the data is a fundamental aspect of nonlinear inversion.

\section{Acknowledgment}

The constructive remarks of the reviewers are greatly appreciated. This research was partly supported by the European Communities (DG XII).

\section{Appendix: the elimination of the quadratic nonlinearities in the Newton-Marchenko method}

The second-order contribution to the solution $K$ of the Newton-Marchenko equation (27) is by analogy with (15b) given by

$K_{2}(\alpha, \hat{\boldsymbol{n}}, \boldsymbol{r})=\int \mathrm{d}^{2} n^{\prime} R_{2}\left(\alpha, \hat{\boldsymbol{n}}, \hat{\boldsymbol{n}}^{\prime}, \boldsymbol{r}\right)+\int_{0}^{\infty} \mathrm{d} \beta \int \mathrm{d}^{2} n^{\prime} R_{1}\left(\alpha+\beta, \hat{\boldsymbol{n}}, \hat{\boldsymbol{n}}^{\prime}, \boldsymbol{r}\right) K_{1}\left(\beta, \hat{\boldsymbol{n}}^{\prime}, \boldsymbol{r}\right)$.

Inserting the second-order Born approximation (30b) in (26) gives

$$
\begin{aligned}
& R_{2}\left(\alpha, \hat{\boldsymbol{n}}, \hat{\boldsymbol{n}}^{\prime}, \boldsymbol{x}\right)=\frac{\mathrm{i}}{64 \pi^{3}} \int_{-\infty}^{\infty} \mathrm{d} k k \int \mathrm{d}^{3} \boldsymbol{r} \int \mathrm{d}^{3} \boldsymbol{r}^{\prime} \frac{V(\boldsymbol{r}) V\left(\boldsymbol{r}^{\prime}\right)}{\left|\boldsymbol{r}-\boldsymbol{r}^{\prime}\right|} \\
& \times \exp \left[\mathrm{i} k \hat{\boldsymbol{n}}^{\prime} \cdot(\boldsymbol{r}-\boldsymbol{x})\right] \exp \left[\mathrm{i} k \hat{\boldsymbol{n}} \cdot\left(r^{\prime}-x\right)\right] \exp \left(\mathrm{i} k\left|\boldsymbol{r}-\boldsymbol{r}^{\prime}\right|\right) \exp (-\mathrm{i} k \alpha) .
\end{aligned}
$$


Carrying out the integration $\int \mathrm{d}^{2} n^{\prime}$ over the unit sphere gives

$$
\begin{aligned}
\int \mathrm{d}^{2} n^{\prime} R_{2}\left(\alpha, \hat{\boldsymbol{n}}, \hat{\boldsymbol{n}}^{\prime}, \boldsymbol{x}\right)=\frac{\mathrm{i}}{16 \pi^{2}} \int_{-\infty}^{\infty} \mathrm{d} k \int \mathrm{d}^{3} r \int \mathrm{d}^{3} \boldsymbol{r}^{\prime} \frac{V(\boldsymbol{r}) V\left(\boldsymbol{r}^{\prime}\right)}{\left|\boldsymbol{r}-\boldsymbol{r}^{\prime}\right|\left|\boldsymbol{r}^{\prime}-\boldsymbol{x}\right|} \\
\times \sin \left(k\left|\boldsymbol{r}^{\prime}-\boldsymbol{x}\right|\right) \exp [\mathrm{i} k \hat{\boldsymbol{n}} \cdot(\boldsymbol{r}-\boldsymbol{x})] \exp \left(\mathrm{i} k \mid \boldsymbol{r}-\boldsymbol{r}^{\prime}\right) \exp (-\mathrm{i} k \alpha) .
\end{aligned}
$$

The second term on the right-hand side of (A1) follows by using (31) for $K_{1}$, and by inserting the first Born approximation (30a) in (26). This gives

$$
\begin{aligned}
\int_{0}^{\infty} \mathrm{d} \beta \int \mathrm{d}^{2} n^{\prime} & R_{1}\left(\alpha+\beta, \hat{\boldsymbol{n}}, \hat{\boldsymbol{n}}^{\prime}, \boldsymbol{x}\right) K_{1}\left(\beta, \hat{\boldsymbol{n}}^{\prime}, \boldsymbol{x}\right)=\frac{-1}{64 \pi^{4}} \int_{0}^{\infty} \mathrm{d} \beta \int \mathrm{d}^{2} n^{\prime} \int_{-\infty}^{\infty} \mathrm{d} k_{1} k_{1} \int \mathrm{d}^{3} r V(\boldsymbol{r}) \\
& \times \exp \left[\mathrm{i} k_{1} \hat{\boldsymbol{n}}^{\prime} \cdot(\boldsymbol{r}-\boldsymbol{x})\right] \exp \left[\mathrm{i} k_{1} \hat{\boldsymbol{n}} \cdot(\boldsymbol{r}-\boldsymbol{x})\right] \exp \left[-\mathrm{i} k_{1}(\alpha+\beta)\right] \int_{-\infty}^{\infty} \mathrm{d} k_{2} \\
& \times \int \mathrm{d}^{3} \boldsymbol{r}^{\prime} V\left(\boldsymbol{r}^{\prime}\right) \frac{\sin \left(k_{2}\left|\boldsymbol{r}^{\prime}-\boldsymbol{x}\right|\right)}{\left|\boldsymbol{r}^{\prime}-\boldsymbol{x}\right|} \exp \left[\mathrm{i} k_{2}\left(\hat{\boldsymbol{n}}^{\prime} \cdot\left(\boldsymbol{r}^{\prime}-\boldsymbol{x}\right)-\beta\right)\right]
\end{aligned}
$$

Expanding the sine in two exponentials, and integrating over $k_{2}$ gives two delta functions, $\delta\left(\beta-\hat{\boldsymbol{n}}^{\prime} \cdot\left(\boldsymbol{r}^{\prime}-\boldsymbol{x}\right)-\left|\boldsymbol{r}^{\prime}-\boldsymbol{x}\right|\right)$ and $\delta\left(\beta-\boldsymbol{n}^{\prime} \cdot\left(\boldsymbol{r}^{\prime}-\boldsymbol{x}\right)+\left|\boldsymbol{r}^{\prime}-\boldsymbol{x}\right|\right)$. The last delta function does not contribute since $\beta>0$ and $\hat{n}^{\prime} \cdot\left(\boldsymbol{r}^{\prime}-\boldsymbol{x}\right)-\left|\boldsymbol{r}^{\prime}-\boldsymbol{x}\right|<0$. This gives

$$
\begin{aligned}
\int_{0}^{\infty} \mathrm{d} \beta \int \mathrm{d}^{2} n^{\prime} R_{1}\left(\alpha+\beta, \hat{\boldsymbol{n}}, \hat{\boldsymbol{n}}^{\prime}, \boldsymbol{x}\right) K_{1}\left(\beta, \hat{\boldsymbol{n}}^{\prime}, \boldsymbol{x}\right)=\frac{-1}{64 \pi^{3} \mathrm{i}} \int_{0}^{\infty} \mathrm{d} \beta \int \mathrm{d}^{2} n^{\prime} \int_{-\infty}^{\infty} \mathrm{d} k_{1} k_{1} \int \mathrm{d}^{3} r V(\boldsymbol{r}) \\
\times \exp \left[\mathrm{i} k_{1} \hat{\boldsymbol{n}}^{\prime} \cdot(\boldsymbol{r}-\boldsymbol{x})\right] \exp \left[\mathrm{i} k_{1} \hat{\boldsymbol{n}} \cdot(\boldsymbol{r}-\boldsymbol{x})\right] \exp \left[-\mathrm{i} k_{1}(\alpha+\beta)\right] \\
\quad \times \int \mathrm{d}^{3} r^{\prime} \frac{V\left(\boldsymbol{r}^{\prime}\right)}{\left|\boldsymbol{r}^{\prime}-\boldsymbol{x}\right|} \delta\left(\beta-\hat{\boldsymbol{n}}^{\prime} \cdot\left(\boldsymbol{r}^{\prime}-\boldsymbol{x}\right)-\left|\boldsymbol{r}^{\prime}-\boldsymbol{x}\right|\right) .
\end{aligned}
$$

Carrying out the integration over $\beta$ and over the unit sphere $\int \mathrm{d}^{2} n^{\prime}$ produces

$$
\begin{aligned}
\int_{0}^{\infty} \mathrm{d} \beta \int \mathrm{d}^{2} n^{\prime} & R_{1}\left(\alpha+\beta, \hat{\boldsymbol{n}}, \hat{\boldsymbol{n}}^{\prime}, \boldsymbol{x}\right) K_{1}\left(\beta, \hat{\boldsymbol{n}}^{\prime}, \boldsymbol{x}\right)=\frac{\mathrm{i}}{16 \pi^{2}} \int_{-\infty}^{\infty} \mathrm{d} k \int \mathrm{d}^{3} r \int \mathrm{d}^{3} \boldsymbol{r}^{\prime} \frac{V(\boldsymbol{r}) V\left(\boldsymbol{r}^{\prime}\right)}{\left|\boldsymbol{r}-\boldsymbol{r}^{\prime}\right|\left|\boldsymbol{r}^{\prime}-\boldsymbol{x}\right|} \\
& \left.\times \sin \left(k \mid \boldsymbol{r}-\boldsymbol{r}^{\prime}\right)\right) \exp [\mathrm{i} k \hat{\boldsymbol{n}} \cdot(\boldsymbol{r}-\boldsymbol{x})] \exp \left(-\mathrm{i} k\left|\boldsymbol{r}^{\prime}-\boldsymbol{x}\right|\right) \exp (-\mathrm{i} k \alpha) .
\end{aligned}
$$

Adding (A3) and (A6), and setting $\alpha=0$, one finds for the total contribution of the second-order contributions:

$$
\begin{aligned}
K_{2}(\alpha=0, \hat{\boldsymbol{n}}, \boldsymbol{x}) & =\frac{\mathrm{i}}{16 \pi^{2}} \int_{-\infty}^{\infty} \mathrm{d} k \int \mathrm{d}^{3} r \int \mathrm{d}^{3} \boldsymbol{r}^{\prime} \frac{V(\boldsymbol{r}) V\left(\boldsymbol{r}^{\prime}\right)}{\left|\boldsymbol{r}-\boldsymbol{r}^{\prime}\right|\left|\boldsymbol{r}^{\prime}-\boldsymbol{x}\right|} \exp [\mathrm{ik} \hat{\boldsymbol{n}} \cdot(\boldsymbol{r}-\boldsymbol{x})] \\
& \left.\times\left[\sin \left(k\left|\boldsymbol{r}^{\prime}-\boldsymbol{x}\right|\right) \exp \left(\mathrm{i} k\left|\boldsymbol{r}-\boldsymbol{r}^{\prime}\right|\right)+\sin \left(k\left|\boldsymbol{r}-\boldsymbol{r}^{\prime}\right|\right) \exp \left(-\mathrm{i} k \mid \boldsymbol{r}^{\prime}-\boldsymbol{x}\right)\right)\right] .
\end{aligned}
$$

Expanding the sines in exponentials one obtains four terms, two of which cancel each other. The remaining terms can be recombined to give

$$
\begin{aligned}
K_{2}(\alpha=0, \hat{\boldsymbol{n}}, \boldsymbol{x}) & =\frac{1}{32 \pi^{2}} \int_{-\infty}^{\infty} \mathrm{d} k \int \mathrm{d}^{3} r \int \mathrm{d}^{3} \boldsymbol{r}^{\prime} \frac{V(\boldsymbol{r}) V\left(\boldsymbol{r}^{\prime}\right)}{\left|\boldsymbol{r}-\boldsymbol{r}^{\prime}\right|\left|\boldsymbol{r}^{\prime}-\boldsymbol{x}\right|} \\
& \times \exp \left[\mathrm{i} k\left(\left|\boldsymbol{r}-\boldsymbol{r}^{\prime}\right|+\left|\boldsymbol{r}^{\prime}-\boldsymbol{x}\right|\right)\right]\{\exp [\mathrm{i} k \hat{\boldsymbol{n}} \cdot(\boldsymbol{r}-\boldsymbol{x})]-[-\hat{\boldsymbol{n}}]\}
\end{aligned}
$$

where $[-\hat{n}]$ stands for the same term with $\hat{n}$ replaced by $-\hat{\boldsymbol{n}}$. Defining $\boldsymbol{r}_{1} \equiv \boldsymbol{r}-\boldsymbol{r}^{\prime}$ and $r_{2} \equiv r^{\prime}-x$, and carrying out the $k$ integration, one finds

$$
\begin{aligned}
K_{2}(\alpha=0, \hat{\boldsymbol{n}}, \boldsymbol{x}) & =\frac{1}{16 \pi} \int \mathrm{d}^{3} r_{1} \int \mathrm{d}^{3} r_{2} \frac{V\left(\boldsymbol{r}_{1}+\boldsymbol{r}_{2}+\boldsymbol{x}\right) V\left(\boldsymbol{r}_{2}+\boldsymbol{x}\right)}{r_{1} r_{2}} \\
& \times\left\{\delta\left(r_{1}+r_{2}+\hat{\boldsymbol{n}} \cdot\left(\boldsymbol{r}_{1}+\boldsymbol{r}_{2}\right)\right)-[-\hat{\boldsymbol{n}}]\right\}
\end{aligned}
$$


Carrying out the $\boldsymbol{r}_{1}$ and $\boldsymbol{r}_{2}$ integrations in polar coordinates, gives with the definitions $u_{1}=\left(\hat{\boldsymbol{n}} \cdot \hat{\boldsymbol{r}}_{1}\right)$ and $u_{2}=\left(\hat{\boldsymbol{n}} \cdot \boldsymbol{r}_{2}\right)$ :

$$
\begin{aligned}
K_{2}(\alpha=0, \hat{\boldsymbol{n}, \boldsymbol{x}}) & =\frac{1}{16 \pi} \int_{0}^{\infty} \mathrm{d} r_{1} \int_{0}^{\infty} \mathrm{d} r_{2} \int_{0}^{2 \pi} \mathrm{d} \phi_{1} \int_{0}^{2 \pi} \mathrm{d} \phi_{2} \int_{-1}^{1} \mathrm{~d} u_{1} \int_{-1}^{1} \mathrm{~d} u_{2} r_{1} r_{2} \\
& \times \frac{V\left(\boldsymbol{r}_{1}+\boldsymbol{r}_{2}+\boldsymbol{x}\right) V\left(\boldsymbol{r}_{2}+\boldsymbol{x}\right)}{r_{1} r_{2}} \\
& \times\left[\delta\left(r_{1}+r_{2}+u_{1} r_{1}+u_{2} r_{2}\right)-\delta\left(r_{1}+r_{2}-u_{1} r_{1}-u_{2} r_{2}\right)\right] .
\end{aligned}
$$

The delta functions give a contribution equal to zero upon integration over the two variables $u_{1}$ and $u_{2}$. This means that

$$
K_{2}(\alpha=0, \hat{n}, x)=0 .
$$

This confirms that in the Newton-Marchenko algorithm the second-order Born approximation is being cancelled by a repeated iteration of the first-order Born approximation.

\section{References}

Agranovich Z S and Marchenko V A 1963 The Inverse Problem of Scattering Theory (New York: Gordon and Breach)

Balanis G N 1972 The plasma inverse problem J. Math. Phys. 13 1001-5

Berryman J G and Greene R R 1980 Discrete inverse methods for elastic waves in layered media Geophysics $45213-33$

Bojarski N N 1980 One-dimensional direct and inverse scattering in causal space Wave Motion $2115-24$

Bregman N D, Chapman C H and Bailey R C 1985 A noniterative procedure for inverting plane-wave reflection data at several angles of incidence using the Ricatti equation Geophys. Prosp. 33 185-200

Bube K P and Burridge R 1983 The one-dimensional inverse problem of reflection seismology SIAM Rev. 25 $497-559$

Burridge R 1980 The Gel'fand-Levitan, the Marchenko and the Gopinath-Sondhi integral equations of inverse scattering theory, regarded in the context of inverse impulse-response problems Wave Motion 2 $305-23$

Carrion P M 1986 On stability of ID exact methods Inverse Problems 2 1-22

Chadan K and Sabatier P C 1989 Inverse Problems in Quantum Scattering Theory (Berlin: Springer) 2nd edn

Cheney M, Rose J H and DeFacio B 1989 A new equation of scattering theory and its use in inverse scattering Wave Motion 11 175-84

Corones J P, Davison M E and Krueger R J 1983 Direct and inverse scattering in the time domain via invariant imbedding equations $J$. Acoust. Soc. Am. 74 1535-41

DeFacio B and Rose J H 1985 Inverse scattering for the non-spherically-symmetric three-dimensional plasma wave equation Phys. Rev. A 31 897-902

Ge D B 1987 An iterative technique in one-dimensional profile inversion Inverse Problems 3 399-406

Gjevik B, Nilsen A and Höyen J 1976 An attempt at the inverse of reflection data Geophys. Prosp. 24 492-505

Koehler F and Taner M T 1977 Direct and inverse problems relating reflection coefficients and reflection response for horizontally layered media Geophysics $\mathbf{4 2}$ 1199-206.

Krueger R T 1981 Numerical aspects of a dissipative inverse problem IEEE Trans. Antennas Propag. AP-29 253-61

Morawetz C S 1981 A formulation for higher-dimensional inverse problems for the wave equation Comput. Math. Appl. 7 319-31

Morawetz C S and Kriegsmann G A 1983 The calculation of an inverse potential problem SIAM J. Appl. Math. 43 844-54

Moses H E 1956 Calculation of the scattering potential from reflection coefficients Phys. Rev. 102 559-67

Newton R G 1980 Inverse scattering II: three dimensions J. Math. Phys. 21 1698-715

- 1981 Inverse scattering III: three dimensions continued J. Math. Phys. 22 2191-200 
Rodberg L S and Thaler R M 1967 Introduction to the Quantum Theory of Scattering (New York: Academic) Rose J H, Cheney M and DeFacio B 1984 The connection between time- and frequency-domain threedimensional inverse scattering methods J. Math. Phys. 25 2995-3000

1985a Three-dimensional inverse scattering: plasma and variable velocity wave equations $J$. Math. Phys. $262803-13$

- 1985b Physical basis of three-dimensional inverse scattering for the plasma wave equation J. Opt. Soc. Am. A 2 1954-7

1986 Determination of the wave field from scattering data Phys. Rev. Lett. 57 783-6

Snieder R 1990 A perturbative analysis of nonlinear inversion Geophys. J. Int. in press

Snieder R and Tarantola A 1989 Imaging of quantum mechanical potentials Phys. Rev. A 39 3303-9 